\title{
Força de preensão palmar em crianças e adolescentes saudáveis
}

Hand strength in healthy children and teenagers

Patrícia Martins de L. S. Moura ${ }^{1}$, Demóstenes Moreira², Ana Paula L. Caixeta ${ }^{3}$

\section{RESUMO}

Objetivo: Revisão de literatura sobre força de preensão palmar em crianças e adolescentes saudáveis na faixa etária de quatro a 18 anos de idade.

Fontes de dados: Medline, Lilacs e SciELO, no período de 2000 a 2007, utilizando-se as palavras-chave: aperto, mão, força, preensão, criança e adolescente.

Síntese dos dados: A importância da mensuração da força de preensão é fornecer um índice objetivo da integridade funcional dos membros superiores. Estudos avaliando a força da preensão palmar vêm sendo descritos desde 1956, porém a maioria dos artigos engloba a população adulta (19 a 44 anos) e adulta média (45 a 64 anos). Somente um artigo foi publicado no Brasil a respeito da força de crianças e adolescentes. Os trabalhos relatados correlacionam a força de preensão palmar e variáveis antropométricas.

Conclusões: Estudos com delineamento adequado e amostras representativas são necessários para avaliar a força da preensão em crianças e adolescentes no Brasil. Observou-se que os meninos têm força de preensão palmar maior que as meninas, principalmente após 11 anos de idade. Os fatores antropométricos como peso e altura foram os que mais significantemente se associaram à força de preensão palmar.

Palavras-chave: força da mão; aperto de mão; criança; adolescente.

\section{ABSTRACT}

Objective: To review the literature regarding hand grip strength in healthy children and teenagers with four to 18 years old.

Data sources: Databases Medline, Lilacs and SciELO were searched for studies published from 2000 to 2007, using the keywords: grasp, hand, strength, grip, child and adolescent.

Data synthesis: Measures of hand grip strength are important to provide an objective index of the functional integrity of the upper limbs. Studies evaluating the hand grip strength have been published since 1956, however the majority of them enrolled adults (19 to 44 years-old) and middle-age ( 45 to 64 years-old) subjects. Only one article was published in Brazil about hand grip strength of children and teenagers. Most studies try to associate hand grip strength and anthropometric variables.

Conclusions: Prospective randomized trials with large number of patients are needed to evaluate the hand grip strength in Brazilian children and teenagers. Boys have hand grip strength greater than girls, especially after 11 years old. The anthropometric factors such as weight and height were significantly associated with hand grip strength.

Key-words: hand strength; grasp; child; adolescent.
${ }^{1}$ Fisioterapeuta, mestre em Ciências da Saúde pela Universidade de Brasília (UnB). Brasília, DF, Brasil

${ }^{2}$ Fisioterapeuta, doutor em Ciências da Saúde pela UnB e docente da Universidade Paulista (Unip). Brasília, DF, Brasil

${ }^{3}$ Fisioterapeuta da Secretaria de Saúde de Brasília e mestranda em Ciências da Saúde da UnB. Brasília, DF, Brasil
Endereço para correspondência:

Patrícia Martins de Lima e Silva Moura

AOS 06 - Bloco A - apto. 304 - Octogonal

CEP 70660-061 - Brasília/DF.

E-mail: mourapat@yahoo.com.br

Recebido em: 10/10/2007

Aprovado em: 5/5/2008 


\section{Introdução}

A mão do homem é dotada de mecanismos extremamente especializados que permitem atividades únicas. Em constante ação, esse órgão sensitivo e motor apresenta grande complexidade de função e importância psicossocioeconômica. Os movimentos da mão são controlados pelo hemisfério cerebral contralateral e sua inervação se origina no plexo braquial, nas raízes de $\mathrm{C} 5$ a $\mathrm{T}^{(1)}$. Todo o membro superior atua em função da mão, que é posicionada para as atividades básicas, lúdicas e profissionais. Desta forma, o homem é o único ser capaz de manipular objetos e de executar trabalhos manuais com equilíbrio, firmeza e versatilidade ${ }^{(2)}$.

Segundo o estudo clássico de Napier ${ }^{(3)}$, o homem apresenta apenas dois padrões básicos de preensão: de força e de precisão. A preensão de precisão é uma forma mais delicada de e se refere a segurar o objeto entre a face palmar ou lateral dos dedos e o polegar oposto. A preensão de força envolve segurar um objeto entre os dedos parcialmente flexionados, em oposição à contrapressão gerada pela palma da mão, a eminência tenar e o segmento distal do polegar. Como o nome sugere, ela favorece o uso total da força ${ }^{(3,4)}$.

Para Magee ${ }^{(5)}$, os estágios da preensão de força são:

- Abertura da mão, que requer a ação simultânea dos músculos intrínsecos da mão e dos músculos extensores longos.

- Fechamento dos quirodáctilos e do polegar para segurar o objeto e adaptar-se à sua forma, o que envolve os músculos flexores intrínsecos e extrínsecos e os músculos opositores.

- Aplicação da força, que varia de acordo com o peso, as características da superfície, a fragilidade e o uso do objeto, envolvendo novamente os músculos flexores intrínsecos e extrínsecos e os músculos opositores.

- Liberação, quando a mão se abre para liberar o objeto e envolve os mesmos músculos utilizados na abertura da mão.

A força de preensão palmar provoca atividade de alto nível do flexor superficial dos dedos, dos interósseos e do flexor profundo dos dedos. A contração do flexor profundo dos dedos exerce tração sobre a fixação proximal do lumbrical. A flexão simultânea das articulações interfalângicas coloca os músculos intrínsecos sob estiramento distal, produzindo assim flexão das articulações metacarpofângicas ${ }^{(6)}$. A força de preensão é um dos elementos básicos para a pesquisa das capacidades manipulativas, de força e de movimento da mão(7).
A importância da mensuração da força de preensão é fornecer um índice objetivo da integridade funcional dos membros superiores. Os dados colhidos auxiliam o médico e o terapeuta a interpretar resultados e a estabelecer metas adequadas de tratamento ${ }^{(8)}$, além da aplicação clínica de avaliação da inabilidade, resposta ao tratamento e avaliação da habilidade de um paciente em retornar às atividades de vida diária ${ }^{(9)}$.

Para Defrasne Ait-Said, Groslambert e Courty ${ }^{(10)}$, a habilidade de pôr em ordem e de discriminar objetos tem um grande impacto no desenvolvimento de uma criança. A infância é a época em que a criança organiza sua percepção da realidade. Percepção significa saber ou interpretar informações; é o processo de organizar informações novas com as já armazenadas, o que leva a um padrão de reação modificado. A habilidade do regulamento motor é componente importante da função, que contribui, por exemplo, para movimentos eficientes e discriminativos da mão. A regulação da força de preensão é componente essencial do desenvolvimento da criança, ao se analisar, por meio visual, os movimentos de abrir, fechar, prender e puxar os objetos com o uso das mãos. Na regulação motora mais tardia, a criança já é capaz de utilizar a força de preensão para executar as atividades cotidianas, tais como prender, segurar, desenhar.

Neste contexto, realizou-se uma revisão de artigos publicados de 2000 a 2007 nas bases de dados Medline, Lilacs e SciELO, utilizando-se as palavras-chave aperto, mão, força, preensão e criança e adolescente, com o objetivo de fazer um levantamento de dados sobre o estudo da força de preensão em crianças e adolescentes.

\section{Síntese dos dados}

Foram encontrados e incluídos no estudo 13 artigos, todos envolvendo crianças a partir de três anos de idade.

Lee-Valkov et al ${ }^{(11)}$ obtiveram dados normativos para a força de preensão palmar em crianças de três $(n=17)$, quatro $(n=24)$ e cinco anos $(n=22)$ de idade. No teste de força de preensão, tais autores utilizaram um dinamômetro em ambas as mãos e observaram que os testes foram significativamente diferentes nas três faixas etárias.

Van Der Beld et a ${ }^{(12)}$ estudaram 67 crianças de quatro a 11 anos de idade com o dinamômetro Jamar ${ }^{\circledR}$ para testar a força de preensão palmar. A finalidade de tal estudo foi avaliar a precisão e a exatidão do dinamômetro em crianças com miopatia (miopatia congênita, miopatia inflamatória e distrofia muscular) diagnosticada por biópsia muscular. Os autores 
concluíram que o dinamômetro é um excelente método para avaliar a precisão da força, porém, não tem acurácia adequada para testar a extensão da miopatia. Dessa forma, os autores consideraram que o dinamômetro não seria um instrumento útil na decisão de indicar uma biopsia muscular.

Defrasne Ait-Said, Groslambert e Courty ${ }^{(10)}$ avaliaram a força de preensão em 37 crianças de três a seis anos de idade. Antes dos testes de força, os autores avaliaram a compreensão das crianças por meio de diferentes imagens que mostravam pacientes executando testes de força de preensão. As modificações da expressão facial, da forma do aperto e da atitude corporal, em virtude do esforço realizado para executar o teste, foram separadas em quatro níveis de compreensão: não difícil, um pouco difícil, difícil e realmente difícil. Os autores concluíram que, ao verem previamente as imagens, as crianças tinham facilidade em executar o teste de força de preensão palmar sem necessidade do comando verbal. Evidenciaram também, neste estudo, que as crianças acima de cinco anos são capazes de executar tais tarefas.

Diversos autores procuraram relacionar características antropométricos como altura, peso, massa corpórea, percentual de gordura, estado nutritivo e até características específicas da mão com as forças de preensão palmar. Sartório et al ${ }^{(13)}$ relacionaram a força de preensão palmar à composição corporal e a outras variáveis antropométricas em 278 crianças italianas de cinco a 15 anos de idade. Foram analisados peso, altura, área de superfície corpórea (BSA), índice de massa corpórea (IMC), porcentagem de gordura corporal (BF) e massa magra corporal (FFM). A diferença na força de preensão só foi significativa entre os sexos quando se referiu aos índices BF e FFM. O aumento da força de preensão após 11 anos de idade foi mais notável nos meninos que nas meninas. Observou-se também que, entre a mão dominante e a não dominante, a diferença média da força na mão dominante foi $10 \%$ maior em todas as idades.

Kenjle et $a l^{(14)}$ avaliaram o teste de preensão palmar juntamente com o estado de nutricional em 787 crianças indianas de seis a dez anos de idade. A força de preensão foi medida na mão dominante e os fatores antropométricos, como altura, peso, circunferência do braço e dobra cutânea do tríceps, foram coletados. Os pesquisadores observaram que os meninos apresentavam maior força de preensão palmar do que as meninas em todas as idades estudadas e que altura, peso, circunferência do braço e dobra cutânea, além da massa gorda corporal, interferiam significativamente na força de preensão palmar.
Clerke, Clerke e Adams ${ }^{(15)}$ avaliaram se a forma da mão influenciava na força de preensão palmar, utilizando o dinamômetro Griptrack ${ }^{\circledast}$ para analisar a força de preensão palmar. Aplicaram o teste em 112 meninas e em 116 meninos adolescentes (entre 13 e 17 anos de idade) e classificaram a forma da mão em: longa, média e quadrada. Tais autores relataram que os meninos eram expressivamente mais forte que as meninas e as mãos dominantes eram significativamente mais fortes que as não dominantes. Entretanto, não observaram diferença significante entre as diversas formas das mãos. Para verificar a confiabilidade do teste de força de preensão, os autores aplicaram o mesmo teste em 74 meninos e 75 meninas previamente estudados, em intervalo médio de 15 dias.

Esteves $e t$ al ${ }^{(16)}$ estudaram mãos de crianças de ambos os sexos com idade entre sete e 14 anos, medindo a força de preensão máxima por meio de mensuração direta e estabeleceram valores de referência de intensidade em relação à idade, lateralidade e sexo. A mão foi ponderada por duas medidas com paquímetro digital, para posterior classificação do tamanho. A força de preensão máxima foi determinada com a utilização de um dinamômetro de resistência elétrica acoplado a um sistema de aquisição de dados. Os autores encontraram diferença significante entre meninos e meninas e entre grupos de 7, 8, 11 e 14 anos. Ao confrontar a preensão versus lateralidade, as diferenças foram notadas nos grupo de 7, 8, 9, 13 e 14 anos. Já em relação ao sexo, a força foi maior no sexo masculino nos grupos de 7 a 11 anos, com diferenças significantes no período puberal, mostrando que o desenvolvimento da força progride conforme aumenta a faixa etária, com maiores diferenças nos períodos de maturação de cada sexo.

Visnapuu e Jürimäe ${ }^{(17)}$ estudaram as dimensões antropométricas da mão e a força de preensão palmar em 189 jogadores de handebol e basquetebol de dez a 17 anos. Os autores afirmaram que o tamanho da mão influenciava na força de preensão e que tanto o tamanho quanto a força melhoram a exatidão do arremesso da bola em ambos os esportes. Foram utilizados três parâmetros antropométricos relacionados à mão: extensão dos dedos, comprimento deles e perímetro da mão. A força de preensão foi medida com a mão dominante, usando-se o dinamômetro Lafayette ${ }^{\circledR}$. Os autores concluíram que os parâmetros antropométricos gerais, como peso e altura, foram mais importantes para dimensionar a força máxima da preensão palmar com exatidão que os específicos.

A maioria dos artigos encontrados nesta pesquisa objetivou criar dados normativos para preensão de força, de precisão 
e de destreza da mão. Alguns pesquisadores tentaram estabelecer dados mundiais conforme a faixa etária estudada ${ }^{(11)}$, enquanto outros estabeleceram dados normativos para seus próprios países.

Ho et $a l^{(18)}$ propuseram dados normativos para medir a força de preensão e de precisão em 2.982 estudantes chineses sadios de 15 a 22 anos de idade. O dinamômetro utilizado foi o $\mathrm{Jamar}^{\circledR}$ e as medidas antropométricas de peso e altura dos pesquisados, bem como a de dominância da mão, foram comparadas. Os autores concluíram que os indivíduos destros contêm, na mão dominante, força de preensão $10 \%$ acima dos canhotos e que os homens possuem a força de preensão superior em $40 \%$ às mulheres.

Smet e Vercammen ${ }^{(19)}$ avaliaram 487 crianças sadias de cinco a 15 anos de idade e elaboraram um banco de dados normativos da força de preensão palmar em crianças, de acordo com sexo, dominância da mão e idade, utilizado o dinamômetro Jamar ${ }^{\circledR}$. Devido ao grande número de crianças examinadas, a aplicação da força foi mensurada somente uma vez. Observou-se que até 12 anos de idade, a força média da preensão de meninos e meninas era semelhante e, após tal idade, a curva de força dos meninos sofreu uma aceleração de $25 \%$ em comparação à das meninas.

Chang ${ }^{(20)}$ analisou o estado atual da força de preensão e de precisão em 4.844 estudantes sadios de sete a 22 anos de idade em uma cidade de Taiwan. O dinamômetro usado foi o Jamar ${ }^{\circledR}$ e fatores como idade, sexo, altura, peso e mão dominante foram analisados. Observou-se que o pico da força de preensão em homens ocorria aos 20 anos e, nas mulheres, aos 17 e que a força de preensão dos estudantes chineses equivale à dos estudantes americanos.

Häger-Ross e Rösblad ${ }^{(21)}$ estabeleceram dados normativos para força de preensão em 530 crianças suecas de quatro a 16 anos de idade, utilizando o dinamômetro Grippit ${ }^{\circledR}$, sustentado por dez segundos. Os autores observaram que a força se mantém semelhante entre meninos e meninas até os dez anos de idade e, depois, os meninos ficam significantemente mais fortes. Além disso, as crianças destras apresentam $10 \%$ mais força com a mão dominante do que as canhotas. Essas, por sua vez, não mostram diferença na intensidade da energia física entre as duas mãos.

Yim, Cho e Lee ${ }^{(22)}$ determinaram valores normativos da força de preensão palmar, da força de precisão e da destreza da mão, analisando 712 crianças coreanas saudáveis entre sete e 12 anos de idade. Para testar a força de preensão e precisão, utilizaram o dinamômetro Jamar ${ }^{\circledR}$ e, para a destreza manual, o Nine-Hole Peg Board. Os autores concluíram que a força de preensão palmar dos meninos era significantemente maior que a das meninas, em todas as faixas etárias pesquisadas. Entretanto, não houve diferença significante entre os gêneros no teste de precisão e no de destreza manual.

Autores como Van Der Beld et al $l^{(12)}$ e Kamieniarz et al ${ }^{(23)}$ testaram a confiabilidade e a validade de testes de preensão de força, sabendo-se que confiabilidade de um instrumento ou de uma mensuração reside na consistência e/ou concordância de informações fornecidas por diferentes examinadores ou em momentos distintos e que validade é a pertinência ou a adequação de determinado método (teste, aparelho) para informar sobre o fenômeno de interesse ${ }^{(24)}$.

Kamieniarz et al ${ }^{(23)}$, pesquisando dois grupos de crianças, verificaram a confiabilidade dos testes de computador para determinar a destreza manual. O primeiro grupo foi constituído por 84 crianças com idade média de 10,5 anos e o segundo por 61 crianças com idade média de 11,2 anos. Tais crianças foram testadas duas vezes, com intervalo de uma semana entre as aferições. Os pesquisadores mostraram que os testes de computador são confiáveis e os aprovaram para a mensuração da destreza manual. O dinamômetro de Jamar ${ }^{\circledR}$ foi o instrumento mais confiável para determinar a destreza manual.

Pode-se observar, diante da revisão realizada, haver necessidade de novos estudos populacionais com amostras representativas para avaliar a força da preensão em crianças e adolescentes saudáveis no Brasil. A padronização desta medida em nosso meio pode se constituir em instrumento importante para auxiliar no seguimento de pacientes pediátricos com artropatias crônicas, miopatias congênitas e miopatias inflamatórias, entre outras.

\section{Conclusões}

Poucas referências bibliográficas foram encontradas na base de dados Medline, Lilacs e SciELO a respeito de estudos da preensão palmar em crianças e adolescentes, com apenas um deles realizado no Brasil e publicado em 2005. Os resultados observados indicam que os meninos têm maior força de preensão do que as meninas, principalmente após 11 anos de idade, atingindo o pico de força máxima aos 19 anos. Os dados antropométricos, como peso e altura, foram os que mais se associaram à força de preensão, diferente do tamanho e da forma da mão. 


\section{Referências bibliográficas}

1. Machado ABM. Neuroanatomia funcional. $2^{a}$ ed. São Paulo: Atheneu; 2000.

2. Abreu LB. Pronto atendimento ao acidentado de mão: considerações gerais, normas de atendimento. São Paulo: IMESP; 1993.

3. Napier Jr. The prehensile movements of human hand. J Bone Joint Surg Br 1956;38-B:902-13.

4. Freitas PP. Reabilitação da mão. São Paulo: Atheneu; 2006.

5. Magee DJ. Avaliação musculoesquelética. $4^{a}$ ed. São Paulo: Manole; 2005.

6. Smith LK, Weiss EL, LehmkuhI LD. Cinesiologia clínica de Brunnstrom. $5^{\mathrm{a}}$ ed. São Paulo: Manole; 1997.

7. Novo Júnior JM, Cliquet Junior A, Gallo Junior L. Considerações preliminares para o projeto de empunhaduras de dinamômetros. Anais do III Fórum Nacional de Ciência em Saúde; 1996 Oct 13-17; Campos do Jordão, Brasil. p. 17-8.

8. Ashton LA, Myers S. Serial grip strength testing: is role in assessment of wrist and hand disability. Internet J Surg [serial on the internet] 2004;5(2) [cited 2008 Mar 27]. Available from: http://www.ispub.com/ostia/index. php?xmlFilePath=journals/ijs/vol5n2/strength.xml

9. Moreira D, Alvarez RR, Godoy JR, Cambraia AN. Abordagem sobre preensão palmar utilizando o dinamômetro Jamar ${ }^{\circledast \text { : }}$ uma revisão de literatura. RBCM 2003;11:95-9.

10. Defrasne Ait-Said E, Groslambert A, Courty D. Validation of a pictorial rating scale for grip strength evaluation in 3- to 6-years-old children. Neurosci Lett 2007;420:150-4.

11. Lee-Valkov PM, Aaron DH, Eladoumikdachi F, Thornby J, Netscher DT. Measuring normal dexterity values in normal 3-, 4-, and 5-years-old children and then relationship with grip and pinch strength. J Hand Ther 2003;16:22-8.

12. van den Beld WA, van der Sanden GA, Sengers RC, Verbeek AL, Gabreëls FJ. Validity and reproducibility of the Jamar dynamometer in children aged 4-11 years. Disabil Rehabil 2006;28:1303-9.

13. Sartorio A, Lafortuna CL, Pogliaghi S, Trecate L. The impact of gender, body dimension and body composition on hand-grip strength in healthy children. $J$ Endocrinol Invest 2002;25:431-5.

14. Kenjle K, Limaye S, Ghugre PS, Udipi SA. Grip strength as an index for assessment of nutritional status of children aged 6-10 years. J Nutr Sci Vitaminol (Tokyo) 2005;51:87-92.

15. Clerke AM, Clerke JP, Adams RD. Effects of hand shape on maximal isometric grip strength and its reliability in teenagers. J Hand Ther 2005;18:19-29.

16. Esteves AC, Reis DC, Caldeira RM, Leite RM, Moro ARP, Borges Júnior NG. Força de preensão, lateralidade, sexo e características antropométricas da mão de crianças em idade escolar. Rev Bras Cineantropom Desempenho Hum 2005;7:69-75.

17. Visnapuu M, Jürimäe T. Handgrip strength and hand dimensions in young handball and basketball players. J Strength Cond Res 2007;21:923-9.

18. Ho RW, Chang SY, Wang CW, Hwang MH. Grip and key pinch strength: norms for 15to 22-years-old Chinese students. Zhonghua Yi Xue Za Zhi (Taipei) 2000;63:21-7.

19. De Smet L, Vercammen A. Grip strength in children. J Pediatr Orthop B 2001;10:352-4.

20. Chang SY. Grip and key pinch strength: norms for 7 to 22 years-old students in Taiwan. Tzuchi Med J 2002;14:241-52.

21. Häger-Ross $C$, Rösblad B. Norms for grip strength in children aged $4-16$ years. Acta Paediatr 2002;91:617-25.

22. Yim SY, Cho JR, Lee IY. Normative data and developmental characteristics of hand function for elementary school children in Suwon area of Korea: grip, pinch and dexterity study. J Korean Med Sci 2003;18:552-8.

23. Kamieniarz M, Stryla W, Kowalska P, Kamieniarz G. Assessment of the children manual dexterity and hand grip strength: test-retest reliability study. Comp Meth Scie Tech 2002;8:69-78.

24. Figueiredo IM, Sampaio RF, Mancini MC, Silva FCM, Souza MAP. Teste de força de preensão utilizando o dinamômetro Jamar. Acta Fisiatr 2007;14:104-10. 